\title{
The regression to right angles tendency, lateral inhibition, and the transversals in the Zöllner and Poggendorff illusions
}

\author{
W. H. N. HOTOPF and S. H. ROBERTSON \\ Department of Social Psychology, London School of Economics, London, WC2A 2AE, England
}

\begin{abstract}
A new version of the Zöllner illusion is demonstrated. Two different ways in which the regression to right angles tendency might operate are distinguished and considered in relation to the illusion. Experiments are reported which show that the one consistent with lateral inhibition between orientation detectors gives the better explanation of the illusion. The implications of this for the Poggendorff illusion are considered.
\end{abstract}

In attempting to devise a form of the Zöllner illusion which would be difficult to explain in terms of Gregory's inappropriate constancy scaling theory of illusion. Hotopf (1966) discovered a new illusion. which is shown in Figure 1. As can be seen, the transversals, particularly the longer ones, though actually straight, appear at their ends to be bent inwards to the vertical. The transversals in Figure 1 are alternately at $30^{\circ}$ and at $40^{\circ}$ to the vertical and other alternations of angle yield similar results, though the ones in Figure 1 seem to be about the most effective. If Figure 1 is rotated through $45^{\circ}$, the Zöllner effect (apparent divergence of the parallel lines) is increased and the bend in the transversals decreased. This appears to be due to the resistance to distortion of horizontal and vertical lines or lines close to these orientations. On the other hand, smudging Figure 1 by Xeroxing it and blowing it up increases the apparent bends in the transversals. The effect can also be demonstrated with a single vertical line crossed by three transversals at $40^{\circ}, 30^{\circ}$, and $40^{\circ}$ to it. as Figure $5 c$ shows. If the transversals are presented by a projector on a screen, and the vertical line on the same screen by a second projector so that the vertical line can be moved across the transversals, it is easy to see the kinks the transversals show changing according to the position of the vertical line. To what is this illusion to be attributed?

We postulate that the illusion is due to the regression to right angles tendency on the part of intersecting lines which change their apparent orientation so as to make the angle they enclose nearer to a right angle. This effect is manifested in "angle"

This work was supported by Grant HR 2604/2 from the Social Science Research Council. The authors would like to thank Mrs. E. Wilson and Mrs. J. Baker of the Geography Department of the London School of Economics for drawing the figures used in this article. Requests for reprints should be sent to W. H. N. Hotopf, Department of Social Psychology, London School of Economics, Houghton Street, London WC2A 2AE, England.

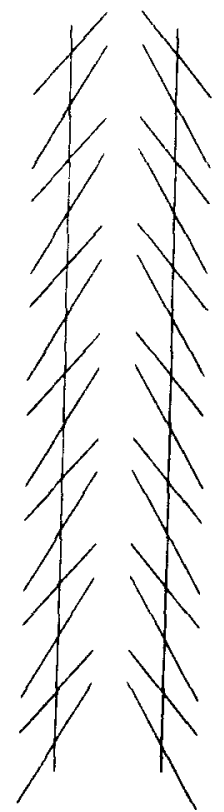

Figure 1. A version of the Zöllner illusion where the transversals appear bent.

illusions such as the Zöllner and the Hering and Wundt figures. Hotopf and Ollerearnshaw (1972a, b) have provided evidence that it also applies to the transversals in the Zöllner and Poggendorff illusions. Two types of theory can be distinguished that suggest ways in which the regression to right angles tendency can come about. We call these one-step theories and two-step theories.

One-step theories are those that account for the "angle" illusions by a single operation, namely lateral inhibition between straight-line orientation detectors in the visual cortex (Andrews, 1965; Blakemore, Carpenter, \& Georgeson, 1970; Bouma \& Andriessen, 1970; Carpenter \& Blakemore, 1973). The detailed case for this type of theory has been put forward by 
the last-mentioned authors, Carpenter and Blakemore. They use two arguments: (1) that proximity in orientation rather than proximity in position is related to the amount of the tendency; and (2) that disinhibition can be demonstrated. We will consider these arguments in turn.

Carpenter and Blakemore measured directly the orientation of one arm of an angle, the standard line. as a function of the orientation of the other arm, which we will call the inducing line, by adjusting the orientation of a comparison line until it appeared parallel to the standard. They found the strongest displacement of the standard from the inducing line occurred when the angle between the two lines was between $10^{\circ}$ and $20^{\circ}$. The next strongest effect occurred when the angle formed by the two lines was an obtuse one of from $160^{\circ}$ and $170^{\circ}$. In the latter case. the two lines were as close to one another in orientation as in the former case, but, since they now formed an obtuse angle, they were much further away from one another in position. Nearness in orientation rather than nearness in position appeared to be the determining factor, and this was shown for all angles between $0^{\circ}$ and $360^{\circ}$, the curves for obtuse angles being roughly inverted mirror images of those for acute angles. Theoretically. this is an important finding that to our knowledge has not been reported elsewhere in the literature. It is based upon the observations of two subjects, themselves the authors
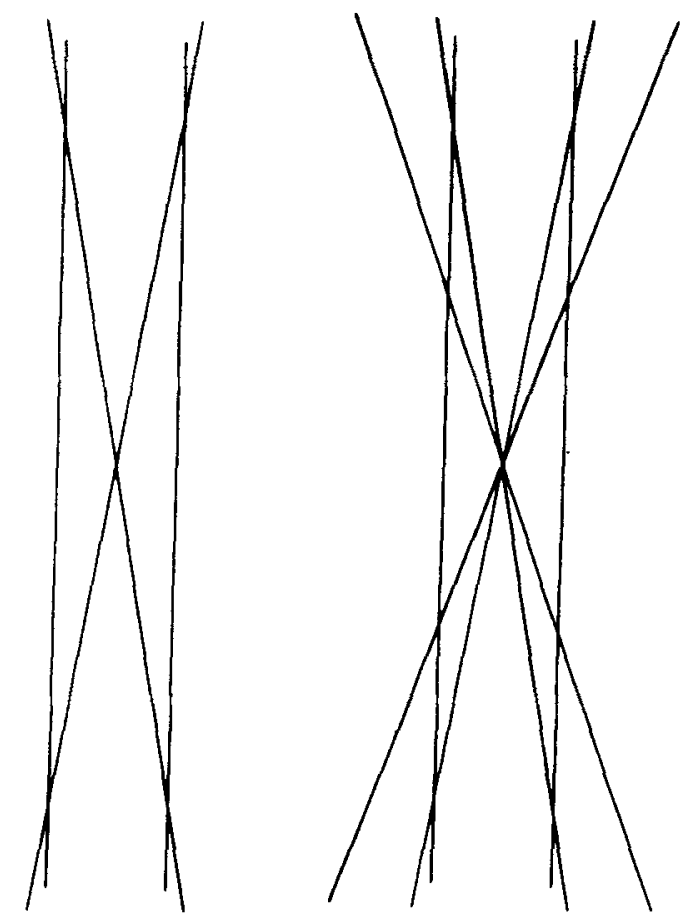

Figure 2. The Hering figure with (left) one pair and (right) two pairs of oblique lines. of the experiment, and though they add that they "confirmed the basic finding informally with several other subjects," they do not say whether these subjects were naive ones.

Carpenter and Blakemore furthermore showed that if for a given angle formed by the standard and the inducing line a third line was introduced, joining the other two at their apex. then this reduced the apparent angular displacement of the standard, depending upon how similar the orientation of the third line was to that of the inducing line. They contended that the third line inhibited the inducing line. thus reducing its inhibition of the standard line. In their experiment, they held the position of the third line constant and found that the greatest disinhibition effect occurred when the angle formed by the third line with the inducing line was between $10^{\circ}$ and $20^{\circ}$. This was in conformity with the results referred to above. They were not, however, able to show anything corresponding to the inverted mirror-image effect when the angle between the third line and the inducing line was increased to a large obtuse angle.

Furthermore, if disinhibiton were a factor, one would not expect certain illusions where the inducing figure is a fan of lines, like the Hering figure, to work as well as they do. Figure 2 demonstrates this by showing that the illusion is not reduced by adding to Figure 2 (left) another pair of oblique lines, as in Figure 2 (right), differing by $10^{\circ}$ from the first pair.

Two-step theories account for the regression to right angles effect by postulating repulsion between the two interacting lines (or attraction when they are close together and repulsion when further away), causing a bend in the lines which, in the case of illusions like the Zöllner and Poggendorff, is by a subsequent process (second step) smoothed away so as to yield straight lines at an angle to one another which is nearer a right angle.

Inevitably it is Helmholtz (1909) who is quoted as first noticing that if the transversals in the Poggendorff illusion are drawn so as to make a long line, then a small tuck in towards the parallel can be discerned just at the point of intersection. To accentuate this effect, we have drawn in Figure 3 a version of the Poggendorff figure where the transversals are not collinear, so that two of these inward tucks at the point of intersection may summate to give the impression of a single transversal, which on meeting the parallels passes horizontally behind them.

A similar effect was studied by Schilder and Wechsler (1936) by means of demonstration figures. In Figures $4 a$ and $4 b$, they point out that where the parallel lines form acute angles with the thick white transversal or black triangle, they appear to bend down slightly in accordance with the regression to right angles tendency. As Figure $4 \mathrm{~b}$ shows, however, they do not do this when the parallel lines are at right 
angles to the black triangle. On the other hand, Figure $4 \mathrm{~b}$ does demonstrate the stepwise appearance of the oblique edge of the triangle, an effect not normally noticed. in the parallels of the Zöllner illusion.

In recent times. Chiang (1968) and Glass (1970) have attributed these effects to blurring in foveal vision from spherical or chromatic aberration and other factors. They have suggested that they may at least in part be responsible for illusions like the Poggendorff illusion. the bent line being seen as a straight one as a result of a "faulty interpretation" by the visual system (Glass) or the operation of some sort of averaging system which operates to take the "line of best fit" "through the asymmetrical pattern of neural excitation (Pressey \& den Heyer, 1968).

On the other hand. Békésy (1967, pp. 230-233) suggested lateral inhibition, operating at peripheral levels, as the explanation of a number of illusions, such as the Mueller-Lyer, though he tested the illusion on the skin, using an acute angled piece of cardboard as the stimulus. His results showed that the point of intersection of the two armis of the angle was displaced in the predicted direction, i.e., to within the area enclosed by the arms of the acute angle, and that the angle was perceived as nearer to a right angle.

The only physiological study of the theory of lateral inhibition known to us is that of Burns and Pritchard (1971), who in a micro-electrode study of neurons in the cat's visual cortex endeavored to find out whether the arms of an acute angle stimulus were changed in orientation, as claimed by Carpenter and Blakemore (1973). or in position. Their results came out in favor of the latter and were formally identical with those obtained by Békésy (cf. their Figures 6 and 7 with Figures 180 and 181 in Békésy's book). They explained them in terms of attraction between the two lines when they were close together and repulsion

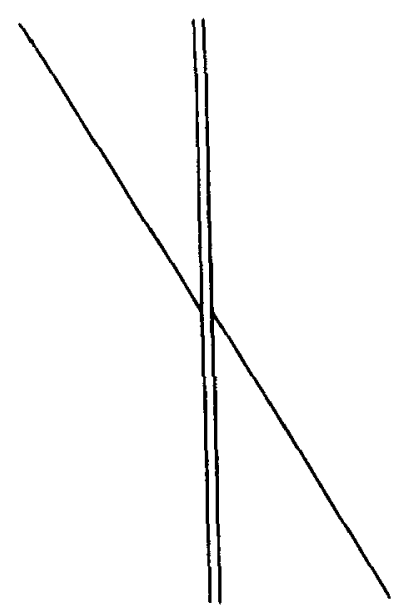

Figure 3. Parallel, but not collinear, transversals in the Poggendorff figure apparently bent inwards at the point of contact.

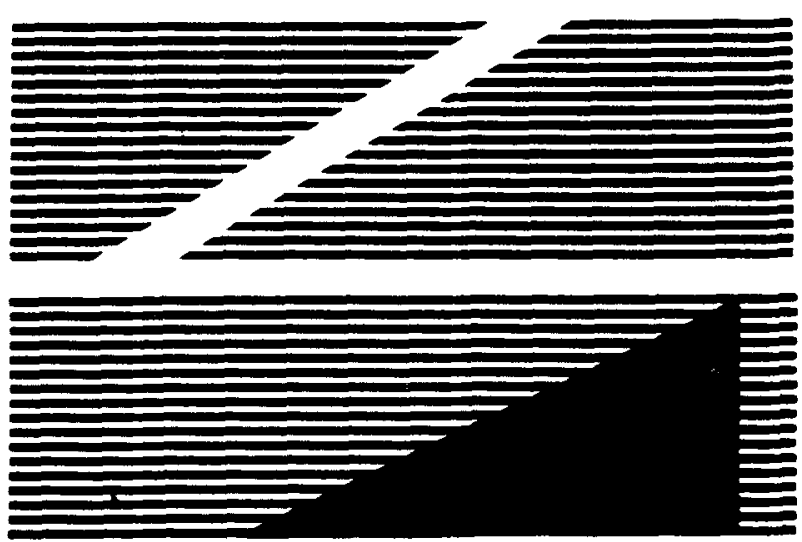

Figure 4. (a) Schilder and Wechulex's demonetration of the clubbing of ends of parallel lines at their point of contact with a white transversal line. (b) Another demontration by Schilder and Wechsler of the clubbing of parallel lines at thet point of contact with a transversal line and the stepwise appearance of the oblique edge of the black triangle. [Figures $4 a$ and $4 b$ are from Schilder $\&$ Wechsler (1936). Copyright 1936 by the American Psychological Association. Reprinted by permission.]

when further apart. They do not, however, appear to allow for the possibility that the lateral inhibition may have operated earlier in the visual system so that the input to the cortical neurons had already the shape they found. But at whatever level the effect operated, if their findings are to account for illusions like the Zöllner and the Poggendorff, then a subsequent process of normalization must be postulated to account for the fact that the lines forming angles in these illusions, as normally drawn, do not appear bent.

We need. however, to account for those cases where the lines do appear bent, i.e., when the normalization process is not working, as shown, for example, in Figures 3 and 4 . The key feature of those figures is that the lines showing the bend were long ones. In such circumstances, there is much more information concerning the true orientation of the line than when it is a short one, and so, instead of the change of orientation near the point of intersection being distributed over the line as a whole, it is confined simply to that space. In the case of the Poggendorff figure, it has long been recognized that the illusion of noncollinearity is considerably reduced when the transversal is a long one, and this would follow from the explanation advanced.

Turning now to the new illusion shown in Figure 1. which of the two types of theory, one-step or two-step, should w'e choose to explain it? The question is rhetorical since the senior author was a two-step theorist from the start and saw this illusion as a means of demonstrating the theory. But let us go through the arguments. One-step theorists could argue that the bends in the $30^{\circ}$ transversals were due to the influence in opposite directions of the regression to right angles 
tendency of the parallels and the $40^{\circ}$ transversals on the $30^{\circ}$ transversals. The parallels would increase the apparent angle of the $30^{\circ}$ transversals with the vertical, while the $40^{\circ}$ transversals would decrease it. Hence the illusion observed in Figure 1 would be caused by a double bend in the $30^{\circ}$ transversals, outward at the point of intersection with the vertical and inward at the ends where nearest to the $40^{\circ}$ transversals. But one would not expect this effect to occur with the $40^{\circ}$ transversals, since here the vertical parallels and the $30^{\circ}$ transversals should operate in the same direction, i.e., away from the vertical. However, in the opinion of many observers, the $40^{\circ}$ transversals show a slight tendency to be distorted in the same way as the $30^{\circ}$ ones. Furthermore, it seems unlikely that the transversals would have much effect on one another in view of the gap separating them. On these grounds, a one-step theory was not entertained. A two-step theory. on the other hand, could argue that the illusion was simply due to the distortion of the transversals by the vertical, according to the regression to right angles tendency, but that this was not normalized because the total figure, unlike the normal Zöllner figure, was too complex for this to occur. This implies that the normalization process is not something operating individually, as though the line of best fit was taken separately for every transversal and for each parallel or for every intersection, but rather that the process was a global one acting on the figure as a whole, or at least that the decisions with regard to the $30^{\circ}$ transversal and vertical were not independent of the decisions for the $40^{\circ}$ transversals and vertical, and conversely. We were left, therefore, with the hypothesis that, in the case of the $30^{\circ}$ transversals, these transversals showed a distortion such that their orientation near the point of intersec. tion with the vertical was greater than $30^{\circ}$ but that at their ends there was no such distortion, i.e., that at their ends they lay in an orientation of $30^{\circ}$. This hypothesis was subjected to experimental test.

\section{EXPERIMENT I}

\section{Method}

Stimuli. Three different types of standard displays were engraved in black on metal plates. These are called Displays A, B, and C and are shown in Figures $5 a, 5 b$, and $5 c$. The first two standard displays were used for control groups in order to determine whether the third, Display $C$, used for the experimental group, caused difficulties in measurement solely by virtue of its complexity. The metal plates were $89 \mathrm{~mm}$ in height and $75.5 \mathrm{~mm}$ wide at the top and $72 \mathrm{~mm}$ at the bottom, being slightly tapered to facilitate insertion into the display stand. The vertical lines in Displays $A$ and $C$ were $50 \mathrm{~mm}$ long, the $30^{\circ}$ transversal in all displays, $31.5 \mathrm{~mm}$ long, and the $40^{\circ}$ transversals in Displays B and C, $25 \mathrm{~mm}$ long. All lines were $0.5 \mathrm{~mm}$ in width. The $30^{\circ}$ transversals bisected the vertical in Displays $A$ and $C$, and the $40^{\circ}$ transversals in Display C crossed the vertical one-quarter and three-quarters of the way up its length. The positions of the lines on the plates were the same in all three displays. Consequently, Displays A and B were identical with a

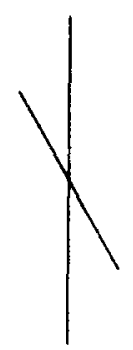

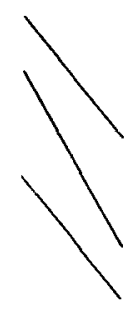

b

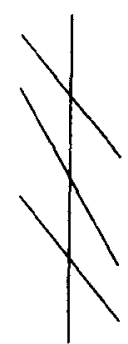

C
Figure 5. Standard displays used in Experiment $I$.

Display C. except for the omission of the $40^{\circ}$ transversals and of the vertical, respectively.

In addition to the standard plates, there were 27 comparison plates. These were of the same dimensions as the standard plates. On each of them there was a line one-third the length of the $30^{\circ}$ transversal on the standards, i.e., $10.5 \mathrm{~mm}$ long, and of the same width, placed in such a way as to be in the same position on the comparison plates as the top, middle, or bottom third of the $30^{\circ}$ transversal on the standard plates. The orientation of each of these thirds of the standard varied in steps of $12^{\circ}$ from $28^{\circ}$ to $32^{\circ}$.

One standard and one comparison plate were placed side by side in a stand which was clamped to a bench in front of which the subject sat. The display stand was tilted at an angle of $677^{\circ}$ to the horizontal. The approximate angle of vision for the subject was $23^{\circ}$ to the horizontal, and the distance, $101.5 \mathrm{~cm}$. The luminance of the stimuli was kept constant through the use of strip lighting and blinds at $1.3 \mathrm{fL}$ for the lines and $4.0 \mathrm{fL}$ for the plates on which they were engraved.

Subjects. The subjects were paid volunteer university students. They were divided in to three groups of 12 subjects each. Each group matched the $30^{\circ}$ transversal on one standard only with the comparison lines. They are called Groups A, B, and C, according to which display they jud ged.

Procedure. Each subject had a 10-min period in which to read the instructions and to ask questions concerning procedure. He was asked to judge comparison lines for each third of the $30^{\circ}$ standard line and to state whether they were steeper, flatter, or of the same orientation. He was shown the standard plate for his group and three comparison plates in turn, one for each third of the standard.

Counterbalancing was adopted such that in each group the six possible orders of presentation of the three segments (top, middle, and bottom) were allocated to two subjects in each case. Further, the order for his counterbalanced condition was repeated for each subject in reverse. Thus, each set of comparisons was done twice. Within each set of comparisons, each of the nine comparison plates was judged twice, once on the left and once on the right of the standard. The order of these 18 presentations was randomized throughout the experiment. Subjects were randomly allocated to groups and counterbalanced conditions with in them as a precaution against any selection, time of day, or other effects that may have been operating.

In all. each subject made 108 judgments -4 for each comparison plate, 36 for each of the three segments of the $30^{\circ}$ standard transversal, and 54 each for right-and left-hand presentation of the comparison plates.

\section{Results}

The PSE was defined as the average angle of the comparison lines for each particular third of the $30^{\circ}$ standard transversal that was judged equal in orientation to the third in question. These are set out 
for each standard in Table 1. Inspection of the table immediately shows that our hypothesis was not supported. As opposed to there being, in the case of the experimental group (Group C), displacement for the middle third so as to increase the angle, and correct judgment for the two outer thirds, we find the reverse has taken place. Though, as predicted, the angle for each third of the $30^{\circ}$ standard transversal in Display A was increased, those in Display B were decreased. Thus, contrary to hypothesis, the $40^{\circ}$ transversals seem to be having an effect on the $30^{\circ}$ one in conformity with the regression to right angles tendency. Display $C$ which combines $A$ and $B$ may be regarded as the sum of the two effects.

It is true that the differences are slight. Nevertheless, they are logical and, in general, statistically significant. Considering first of all the differences in angles between the different thirds within each group and using a t test for correlated means, none of the differences between the thirds of Display A or Display B was significant. As regards Display $C$, the differences between the middle third, on the one hand, and the top and bottom thirds, on the other, were both in the right direction, although only the former was significant ( $p<.005$, one-tailed). There was also a significant difference between the top and bottom thirds of Display $C \quad(p<.05$, two-tailed), which we had not predicted and which we cannot explain.

The observed distortions in Groups A and B were significantly in the direction of the regression to right angle effect. Pooling the data for each group and comparing the average angle with a hypothesized average of $30^{\circ}, p<.0005$ in the case of Group $A$ and $p<.005$ in that of Group B, one-tailed in both cases. Finally, the average angles of the top, middle, and bottom thirds of Group B differed in each case from the average angles of the respective third of Group $A$ according to $t$ tests for uncorrelated means ( $p<.0005$, in each case, one-tailed). On the other hand, comparing Group C with Group B and using the same test, there was a significant difference between the two groups only in the case of the middle third ( $p<.05$. one-tailed).

\section{Discussion}

Though the data do not require the postulation of a normalizing process, it is noticeable that whereas the effect of the vertical on its own on the $30^{\circ}$ transversal was stronger than that of the two $40^{\circ}$ transversals on their own, when these were combined the effect of the $40^{\circ}$ transversals on the top and bottom thirds remained unchanged and their effect on the middle third seemed stronger than that of the vertical. Could it be that there is still some normalizing process at work so that we tend to see this line as straight, in accordance with the line of best fit, and that this is determined by the two ends of the line affecting the
Table 1

PSEs and Cell Variances for Each Third of the 30-Deg Transversal in the Different Displays

\begin{tabular}{|c|c|c|c|c|c|c|}
\hline \multirow[b]{3}{*}{ Display } & \multicolumn{6}{|c|}{ Third Under Consideration } \\
\hline & \multicolumn{2}{|c|}{ Top } & \multicolumn{2}{|c|}{ Middle } & \multicolumn{2}{|c|}{ Bottom } \\
\hline & $\begin{array}{c}\text { PSE } \\
\text { (Deg) }\end{array}$ & $\begin{array}{c}\text { Variance } \\
\text { (Deg) }\end{array}$ & $\begin{array}{c}\text { PSE } \\
\text { (Deg) }\end{array}$ & $\begin{array}{c}\text { Variance } \\
\text { (Deg) }\end{array}$ & $\begin{array}{c}\text { PSE } \\
\text { (Deg) }\end{array}$ & $\begin{array}{c}\text { Variance } \\
\text { (Deg) }\end{array}$ \\
\hline $\mathbf{A}$ & 30.33 & .14 & 30.55 & .18 & 30.47 & .19 \\
\hline B & 29.84 & .25 & 29.78 & .14 & 29.80 & .10 \\
\hline $\mathrm{C}$ & 29.67 & .07 & 30.04 & .14 & 29.88 & .03 \\
\hline
\end{tabular}

middle section? To decide this, a second experiment was carried out identical to the first except that only the middle third of the $30^{\circ}$ transversal was displayed.

\section{EXPERIMENT II}

\section{Method}

Stimuli. The stimuli were the same as in the previous experiment, except that the $30^{\circ}$ transversal in Displays A, B, and C of Figure 5 was only $10.5 \mathrm{~mm}$ in length. It occupied the same relative position to the other lines and to the plates on which they were engraved as did the middle third in the previous experiment. Similarly, only the nine comparison plates of the middle third from the previous experiment were used.

Subjects. There were 12 subjects, who were paid volunteer university students.

Procedure. The same procedure was used as in the previous experiment, the only substantial difference being that each subject judged the single standard in each of the three displays.

\section{Results}

The average PSEs for Displays $\mathrm{A}, \mathrm{B}$, and $\mathrm{C}$ were $30.39^{\circ}, 29.50^{\circ}$, and $29.98^{\circ}$, respectively. The averages in the case of Displays A and B departed significantly from a hypothesized average of $30^{\circ}$ in the direction of the regression to right angles effect $(p<.05$ and $p<.025$, respectively, one-tailed), while this was clearly not the case with Display C. Using one-tailed $t$ tests for correlated means, the differences between Displays $A$ and $C(p<.0025)$ were significant, and so were those between Displays $B$ and $C(p<.01)$. The results, then, are substantially the same as those in Experiment I. Once again, the postulation of a normalizing mechanism, a contextual effect tending to produce a straight line in accordance with the Gestalt law of continuity, is not supported.

\section{DISCUSSION}

Experiments I and II each show that we do not need to postulate a two-step theory to account for the illusion shown in Figure 1. The distortion effects found in Displays A and B apply to the $30^{\circ}$ transversal line as a whole, since no significant differences in slope were found in Experiment I between the different thirds for each display. This supports the 


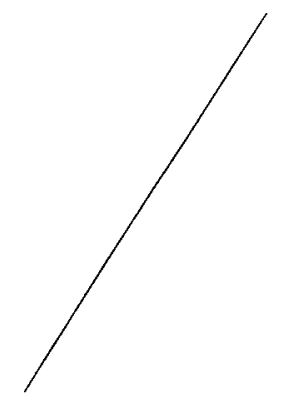

Figure 6. Oblique line with top and bottom thirds at $2912^{\circ}$ and middle third at $30^{\circ}$ to the vertical.

notion of lateral inhibition operating between orientation detectors. The results with Display $\mathrm{C}$ are also consistent with it. There are, however, two anomalies. We shall consider these briefly and then discuss what we consider to be the main theoretical signiticance of the new illusion.

The first anomaly lies in the failure of the middle third in Display $C$ to be seen as distorted. This cannot be due to disinhibition, i.e., to the $40^{\circ}$ transversals inhibiting the vertical so that the vertical did not inhibit the $30^{\circ}$ transversal, since such an effect would work both ways and there should then have been no distortion of the ends of the $30^{\circ}$ transversal either. An explanation consistent with the one-step theory would be that due to lateral inhibition of the $30^{\circ}$ transversal by both the vertical and the $40^{\circ}$ transversal lines, there would be maximal firing not by neurons responding best to an orientation of $30^{\circ}$ but by neurons responding best to orientations both just above and just below that orientation. A detector unit scanning these neurons would, however, average these two peaks of firing and would thus code the $30^{\circ}$ orientation actually observed.

The second anomaly is that of the appearance of the $40^{\circ}$ transversals. The apparent inward bend of the ends of these lines could be made consistent with a lateral inhibition of orientation detectors theory if it were postulated that the effect of the verticals on them was stronger than that of the $30^{\circ}$ transversals. We have not attempted to measure this, because the illusion with the $40^{\circ}$ transversals is very slight and our method is unlikely to be sensitive enough to pick it up. We must also admit that our explanation is inconsistent with what we found for Display $\mathrm{C}$ with the $30^{\circ}$ transversals, where the $40^{\circ}$ transversals exerted a greater influence than the vertical.

Turning now to the illusion itself, its main significance lies in its demonstration of the regression to right angles effect. Hotopf and Ollerearnshaw (1972a) argued that in the Zöllner illusion there were two illusions, only one of which, the failure of the parallel lines to be seen as parallel, was directly revealed. The other, the illusory orientation of the transversals, they were able to show by experimental means. This is important because of its implication for the Poggendorff illusion, for which they also revealed a distortion of the transversal. That the regression to right angles illusion is a significant component in the Poggendorff illusion has, however, been denied by Day and Dickinson (in press), Pressey and Sweeney (1972), and Weintraub and Tong (1974). Weintraub and Tong quote Hotopf and Ollerearnshaw $(1972 a, b)$ as supporting their view that the regression to right angles tendency has only a small effect. Now, it is true that the first series of experiments (Hotopf \& Ollerearnshaw, 1972a) using the method of constant stimuli, to which Weintraub and Tong are referring, did reveal only a small effect. But this was done, to use Weintraub's terminology, with only a truncated version of the Poggendorff figure, consisting of just a vertical line and a transversal, and using the same rather insensitive method as in the present experiments, where measurement of orientation depended upon judgments of equality, i.e., parallelism matches, only. But when the whole Poggendorff figure was displayed and the method of adjustment was used (Hotopf \& Ollerearnshaw, 1972b), then the amount of change was much larger, being $2.5^{\circ}$ in one experiment and $1.45^{\circ}$ in another. This constituted, in the two different experiments, between a half and third of the total amount of Poggendorff illusion as measured by judgments of collinearity, which may surely be called a signiticant component of the Poggendorff illusion. To deny this would be to separate the Poggendorff from the other angle illusions, all of which directly manifest the regression to right angles effect from which Weintraub and Tong and others so oddly wish to exclude the Poggendorff. Now the virtue of Figure 1 is that it also directly manifests the regression to right angles tendency. Yet, might it not be said that the results of Experiments I and II confirm Weintraub and Tong's criticism, since they

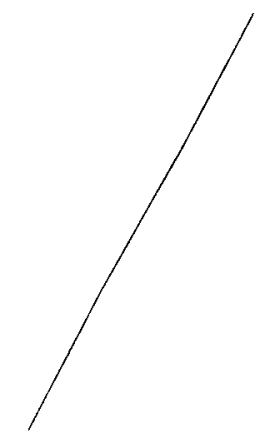

Figure 7. Oblique line with top and bottom thirds at $28^{\circ}$ and middle third at $30^{\circ}$ to the vertical. 
show the effect to be very slight indeed? The answer we must give to this is that our experiments certainly underestimate the extent of the effect. In Figure 6, we have a line the middle section of which is in an orientation of $30^{\circ}$ to the vertical and the ends of which are at $2912^{\circ}$ to the vertical. It is clear that it is impossible to detect this difference of $1 / 2^{\circ}$. Indeed, the figure reveals an interesting limitation in the power of the eye to detect differences in orientation. But what is shown by Figure 6 stands in marked contrast to the differences that are readily observed in Figure 1 or in Figure 5c. And this is so even when we draw the thirds of an oblique line in such a way that there is a difference as large as $2^{\circ}$. This is shown in Figure 7 , where the middle third is at $30^{\circ}$ to the vertical and the two outer thirds are at $28^{\circ}$ to it. Of course, the shape of the distortion is different from that observed in Figures 1 and $5 c$, where the change in angle appears to be gradual and starting from the point of intersection with the vertical. Possibly this makes it easier to notice. What, however, seems to be established is that the regression to right angles tendency and perhaps, therefore, lateral inhibition plays a significant part as one of the components of the Poggendorff illusion.

\section{REFERENCES}

Andrews. D. P. Perception of contours in the central fovea. Nature, 1965, 205. 1218-1220.

BÉKÉsY, G. v. Sensory inhibition. Princeton: University Press, 1967.

Blakemore, C., Carpenter, R. H. S., \& Georgeson, M. A. Lateral inhibition between orientation detectors in the human visual system. Nature, $1970,228,37-39$.

Bouma, H., \& Andriessen, J. J. Induced changes in the perceived orientation of line segments. Vision Research, 1970. 10, 333-349.
Burns, B. D., \& Prurchard, R. Geometrical illusions and the response of neurones in the cat's visual cortex to angle patterns. Journal of Physiology, 1971, 213, 599-616.

Carpenter, R. H. S., \& Blakemore, C. Interactions between orientations in human vision. Experimental Brain Research, 1973, 18, 287-303.

Chiang, C. A new theory to explain geometrical illusions produced by crossing lines. Perception \& Psychophysics, $1968,4,174-176$.

DAY, R. H., \& Dickinson, R. G. The components of the Poggendorff illusion. British Journal of Psychology, in press.

GLASS, L. Effect of blurring on perception of a simple geometric pattern. Nature, 1970, 228, 1341-1342.

HELMHOLTz, H. v. Handbuch der physiologischen Optik (3rd ed.). Hamburg and Leipzig: Voss, 1909-1911. (First published 1856-1866; 2nd ed., 1896.)

Ноторғ, W. H. N. The size constancy theory of visual illusions. British Journal of Psychology, 1966, 57, 307-318.

Hotopf, W. H. N., \& Ollerearnshaw, C. The regression to right angles tendency and the Poggendorff illusion. I. British Journal of Psychology, 1972, 63, 359-367. (a)

Hotopf, W. H. N., \& Ollerearnshaw, C. The regression to right angles tendency and the Poggendorff illusion. II. British Journal of Psychology, 1972, 63, 369-379. (b)

Pressey, A. W., \& Den Heyer, K. Observations on Chiang's 'new' theory of geometrical illusions. Perception \& Psychophysics, 1968, 4, 313-314.

Pressey, A. W., \& Sweeney, $O$. Acute angles and the Poggendorff illusion. Quarterly Joumal of Experimental Psychology, 1972. 24, 169-174.

SCHILdER, P., \& WechsLer, D. The illusion of the oblique intercept. Journal of Experimental Psychology, 1936, 19. 747-757.

Weintraub, D. J., \& Tong, L. Assessing Poggendorff effects via collinearity, perpendicularity, parallelism and Oppe] (distance) experiments. Perception \& Psychophysics, 1974, 16, 213-221.

(Received for publication September 20, 1974; revision received July 1,1975 .) 